\title{
Periodic Gait Plans for Locomotion on Natural Terrain by a Submersible Walking Dredger/ Miner
}

\author{
Dr. Ir. Sritama Sarkar ${ }^{1}$, Dr. Neil Bose ${ }^{2}$, Mr. Mridul K Sarkar ${ }^{3}$ \\ ${ }^{1}$ Project Engineer, Soil Machine Dynamics Ltd. \\ Wincomblee Road, Newcastle Upon Tyne, NE6 3QS, UK \\ sritamasarkar@hotmail.com \\ ${ }^{2}$ Canada Research Chair in Offshore and Underwater Vehicles Design, Memorial University of Newfoundland; Faculty of Engineering and \\ Applied Sciences, \\ Memorial University of Newfoundland, St. John's, Canada A1B 3X5; Tel: +1 (709) 737 4058, Fax: +1 (709) 737 6184, nbose@engr.mun.ca
}

${ }^{3}$ Managing Director, Excavation \& Equipment Manufacturing (P) Ltd., India, WIB (R ), 8/1, Phase IV, Golf Green, Kolkata- 700 095, India; Tel: + 91 (0) 33 24737169; Fax: +91 (0) 33 22219350, mridulsarkar20@,hotmail.com

\begin{abstract}
Use of surface floating dredgers in deep inland reservoirs and continental shelves, either for dredging or mining is restricted by several operational limitations and mobilization problems. Ladder length is a major constraint and in the absence of good approach roads, transportation of bigger surface floating dredgers to inland areas is difficult. In shelf areas, dredging operation is less precise due to currents, waves and winds. Use of small, modular comparatively less expensive submersible dredgers/ miners is a suitable solution to some of the above-mentioned problems. A collaborative research project between Memorial University of Newfoundland, Canada and Excavation \& Equipment Manufacturing (P) Ltd., India was set up to develop, design, build and test a small prototype of a modular walking submersible dredger suitable for deep inland reservoirs. The full-scale electrohydraulically operated prototype was remotely operated on land for all the full-scale prototype tests. This paper describes the theoretical gait plans developed for the designed vehicle to perform straight line and curvilinear locomotion on natural terrain. Representative results showing the comparisons between the theoretical and experimental results for the gait plans are presented.
\end{abstract}

\section{INTRODUCTION}

The use of surface floating dredgers in deep inland reservoirs and continental shelves, either for dredging or mining purposes, is restricted by several operational limitations. Use of smaller surface floating dredgers in deep inland reservoirs is constrained by the ladder (a long truss like structure 'arm' that supports the excavation tool) length. Bigger dredgers have operational restrictions and mobilization problems. In shelf areas, the dredging operation is less precise due to currents, winds and waves. The floating pipelines, floats and winch wires are obstacles to navigational paths for other surface vessels. High investment costs are involved in the construction of bigger high capacity dredgers. It is difficult to modify such systems once they are constructed. The limitation of the existing technology served as the main motivation to design an active legged submersible dredger/ miner, which is described in this paper. The designed vehicle is named the 'Golden Tortoise' because it simulates the belly crawling motion of a tortoise or turtle. A full-scale prototype vehicle was manufactured by Excavation \& Equipment Manufacturing (P) Ltd., (or EEM (P) Ltd.) India. The prototype vehicle is suitable for operation in deep inland reservoirs up to a depth of $50 \mathrm{~m}$ and is designed to excavate sand, silt or clay mixtures in various proportions. The prototype vehicle is shown in Figure1.

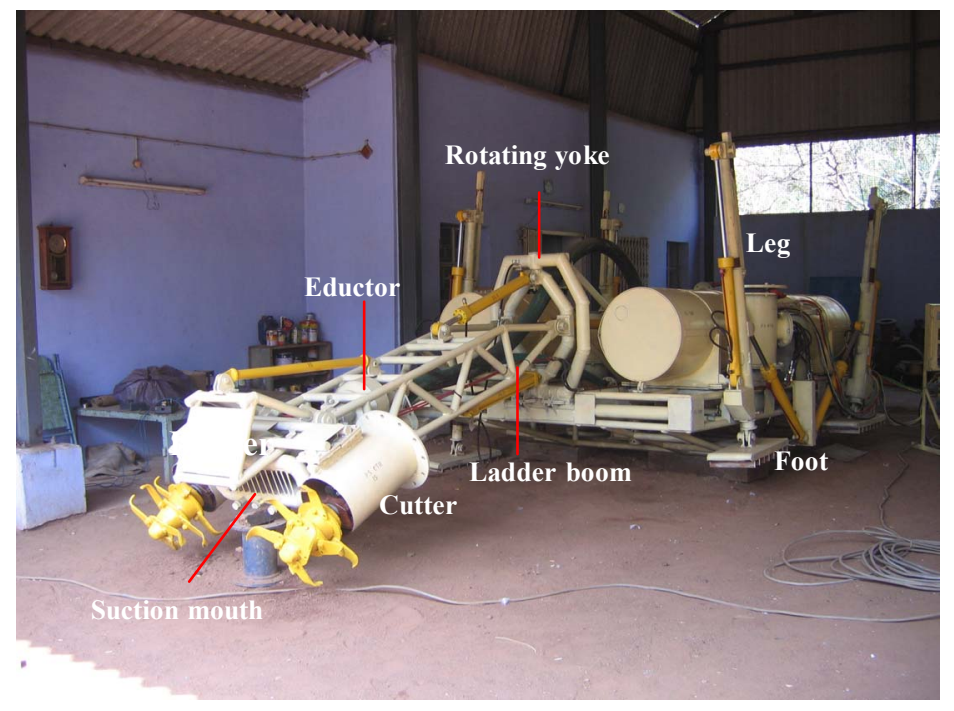

Figure 1. Prototype vehicle "Golden Tortoise" 


\section{A. Prototype Vehicle}

The prototype vehicle is $3.3 \mathrm{~m}$ in length (without the ladder assembly), $2.4 \mathrm{~m}$ in width and $1.8 \mathrm{~m}$ in height. The hull consists of the main body frame, where modular dry pressure hulls having either vertical or horizontal orientations are attached. The ballast tanks, the hydraulic modules and the electric-electronics modules are dry pressure hulls. The same arrangement of the ballast tanks, the hydraulic module and the electric-electronics module are repeated on either side of the designed vehicle. Individual pressure hulls are mounted on resilient material for reducing the vibrations. This also helps in the segregation of vibrations of individual pressure hulls.

The designed vehicle has four ballast tanks attached at the four corners of the vehicle. The ballast tanks will help the vehicle to sink and float during launch and recovery operations. The main function of the ballast tanks is to control the bottom load by adjusting the amount of ballast water. Each ballast tank needs to be controlled separately. The load at each leg determines the soil failure criteria and also balances the operational forces thus preventing slippage and overturning of the vehicle during operation. Hydraulics and electricelectronics modules are present on either side of the designed vehicle.

The excavation system includes a ladder assembly and two interchangeable mechanical drum cutters positioned on either side of the suction mouth. The ladder assembly consisting of a boom and a dipper has three degrees of freedom, including a swing motion and a lifting/ lowering motion of the boom and the dipper. The ladder is attached to a rotating yoke, which is fixed to the main body frame.

A centrifugal dredge pump with an assisting annular eductor pump will be used to transport the excavated material to the surface by pipelines attached to the delivery end of the centrifugal dredge pump. Four hydraulically operated legs with pinned feet are used to perform locomotion on natural terrain. Legs are pinned to the main body frame and thus sudden loads from the legs are transferred to the main body frame and not the dry pressure hulls.

The processing and computational tasks for the movement and operation of the submersible legged dredger/ miner will be distributed between onboard and off board components depending upon the complexity and the time critical factors of the desired tasks. Presently a main power supply line (440 V $\mathrm{AC}, 50 \mathrm{~Hz}$ cycle) is used for the primary power to drive the secondary hydraulic drives. During operation under water, primary electrical power supplied through the umbilical connected to the electrical module of the designed vehicle will drive the secondary hydraulic circuits.

Two different modes of locomotion were proposed for the designed vehicle 1) legged locomotion and 2) belly sliding. During legged locomotion, the designed vehicle rests on its belly before the commencement of the locomotion cycle and at the end of the locomotion cycle. The vehicle body is lifted off the ground and moved forward through a desired distance by the four legs during the locomotion cycle. During the locomotion cycle the vehicle body is supported on the four feet.

In belly sliding mode, the vehicle belly is in constant contact with the ground during the entire locomotion cycle. The belly thus always supports the vehicle weight. The sliding motion of the vehicle belly can be generated by the swinging motions of the four legs, in which case a very small portion of the vehicle weight is supported by the four feet. Alternatively, the sliding motion of the belly can be achieved by anchoring the ladder assembly with the cutters and then swinging the ladder assembly. The swinging of the ladder results in the sliding of the belly in the opposite direction exhibiting a turning behavior of the vehicle around the pivot point defined by the position of the cutters. When the belly sliding is achieved by swinging of the ladder assembly, the four legs are lifted off the ground. Belly sliding is especially effective in very soft soil conditions, where sufficient soil bearing capacity may not be available.

The matrix between the mode and type of locomotion and the terrain type is shown in Table 1.

TABLE I

LOCOMOTION VS. TERRAIN TYPE

\begin{tabular}{|c|c|c|c|c|}
\hline Locomotion & $\begin{array}{c}\text { Level- } \\
\text { Reasonably } \\
\text { flat }\end{array}$ & $\begin{array}{c}\text { Level- } \\
\text { Undulating }\end{array}$ & $\begin{array}{c}\text { Sloped - } \\
\text { Reasonably } \\
\text { flat }\end{array}$ & $\begin{array}{c}\text { Sloped - } \\
\text { Undulating }\end{array}$ \\
\hline $\begin{array}{c}\text { Belly } \\
\text { sliding- } \\
\text { Straight line } \\
\text { locomotion }\end{array}$ & & & & \\
\hline $\begin{array}{c}\text { Belly } \\
\text { sliding- }\end{array}$ & & & & \\
Curvilinear & & & & \\
locomotion & & & & \\
\hline Legged & & & & \\
locomotion & & & & \\
- Straight & & & \\
line & & & & \\
locomotion & & & & \\
\hline Legged & & & & \\
locomotion & & & \\
- & & & \\
Curvilinear & & & \\
locomotion & & & & \\
\hline
\end{tabular}

Shaded boxes in Table 1 indicate that the particular mode and the type of locomotion are possible in the given terrain.

\section{B. Mode of Locomotion}

A unique method of locomotion was developed for the designed vehicle, which solves various problems of other legged locomotion. The various steps of the locomotion cycle are shown in Figure 2.

This periodic gait can be used for straight line and curvilinear locomotion on any terrain. The 'support phase' and the 'transfer phase' of each leg coincide with one another. The transfer phases of the legs start when the vehicle is supported on its belly. The steps shown in Figure 2 are followed for all successive locomotion cycles, but the swing angles of the legs 
may vary depending upon the required step size of the individual legs and that of the vehicle. The gait is thus defined as a non-uniform periodic gait.

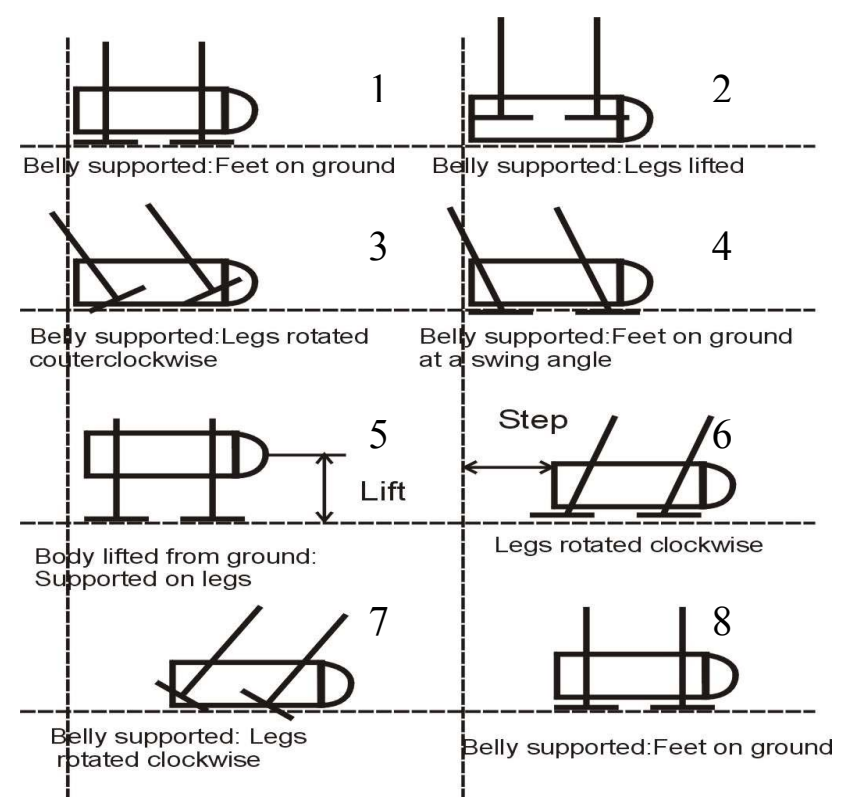

Figure 2. Method of locomotion

The static stability margin is not a major concern for the proposed mode of locomotion as the vehicle is always supported on the four legs. The center of gravity of the designed vehicle is moving together with the legs. The ground reactions conveyed through the legs are transmitted to the main body frame at the leg pin joints, which are responsible for the vehicle movement. The vehicle rests on its belly when the position of the center of gravity due to dredging/ mining has been changed.

With the designed leg linkage, individual leg movements are possible, which allows the designed vehicle to traverse on uneven terrains. The designed vehicle body can be kept horizontal by individual movements of the legs, which is necessary in order to move the ladder assembly and the cutters along the desired trajectory. Forward and backward motions and obstacle avoidances are possible with the leg linkage. For obstacle avoidance 1) the foot can be placed in a different place, 2) the vehicle can be lifted off the ground with the aid of the legs, and 3) thrusters can be used to help the vehicle in swimming.

The feet positions do not change during the vehicle frame movement (Figure 2). This facilitates easy computations of the joint parameters for the feet placement (inverse kinematics) and easy control, essential for any submersible walking dredger/ miner. During successive locomotion cycles the deviation from the proposed path of locomotion can be corrected at the end of each locomotion cycle, as continuous locomotion is not envisaged. No dredging or mining activities are executed during a locomotion cycle. During a dredging cycle the vehicle is either supported on its belly or on its four legs depending on the soil conditions. During the support phases of the legs, the load incident at each leg determines the soil reaction and thus the stability of the vehicle in terms of the soil bearing capacity. The area of contact and the soil reaction increases when the vehicle is supported on its belly.

\section{Theoretical Gait Plan for Straight Line Locomotion}

The magnitude of the step size of individual leg determines whether the designed vehicle will exhibit straight line or curvilinear locomotion. The step sizes of all the legs and the center of gravity of the designed vehicle must be equal for straight-line locomotion. A step generator was developed to predict the step size of the leg as a function of the leg joint parameters. The step generator was further modified to accommodate the level difference due to terrain or submergence at the foot/ soil interface. The slip action at the foot/ soil interface was also considered. The slip for the designed vehicle is due to the shearing action at the foot/ soil interface and not due to the frictional force as is common in other legged vehicles. This is because the feet are kept fixed during the entire locomotion cycle for the designed vehicle as opposed to other legged vehicles.

The step generator for the designed legged vehicle is shown in Figure 3.

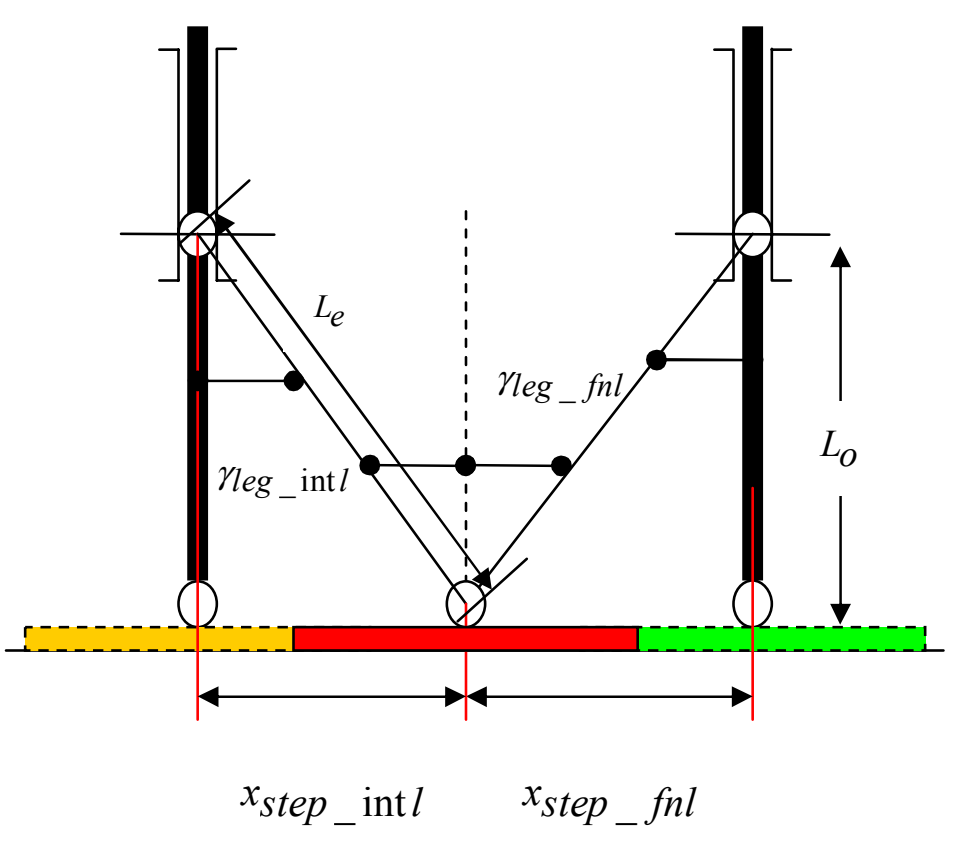

Figure 3. Step generator

The step generator without any level difference, submergence or slip at the foot/ soil interface is deduced as follows,

$x_{\text {step_intl }}=L_{O} * \tan \left(\gamma_{l e g} \_\right.$intl $)$ 
$x_{\text {step_f } f n l}=L_{O} * \tan \left(\gamma_{l e g} f n l\right)$

where, $L_{O}$ is the perpendicular distance between the leg pin center and the foot hinge pine center $(\mathrm{m}), x_{\text {step_intl }}$ is the step size due to initial swing angle of the leg (m), $x_{\text {step }} f n l$ is the step size due to the final swing angle of the leg $(\mathrm{m}), x_{\text {step }}$ is the total step size of individual leg and is the sum of $x_{\text {step_intl }}$ and $x_{\text {step }} f n l(\mathrm{~m}), \gamma_{\text {leg_intl }}$ is the initial angle of leg swing (degree), and $\gamma_{l e g} f n l$ is the final angle of leg swing (degree). Unless otherwise stated, the term 'swing angle' for leg represents the 'half swing angle' of the leg i.e. either $\gamma_{l e g \_i n t l}$ or $\gamma_{l e g} f n l$. The sum of the initial and final swing angle is denoted as the 'total swing angle'.

If two legs are present at two different levels denoted by $L_{1}$ and $L_{2}(\mathrm{~m})$, with a level difference of $(+) \Delta h_{l e g}(\mathrm{~m})$, the modified step generator is given as,

$x_{\text {step_intl }}=x_{\text {step_fnl }}=L_{1} * \tan \left(\gamma_{l e g}\right.$ intl_1 $)$

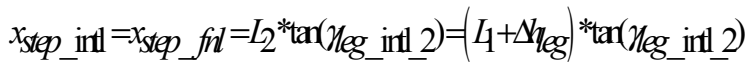

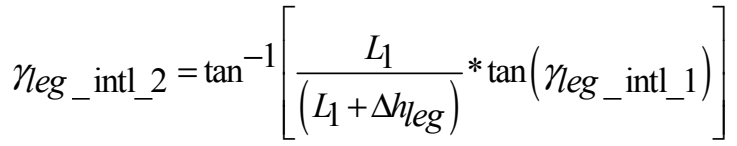

where, the subscripts 1 and 2 represent two different legs. If there is submergence of $\Delta h_{\text {foot }}(\mathrm{m})$ at any foot/ soil interface, the term $\Delta h_{\text {leg }} \quad(\mathrm{m}) \quad$ must be replaced by $\left(\Delta h_{\text {leg }}+\Delta h_{\text {foot }}\right)(\mathrm{m})$ in the above equations.

When there is a slip of $\Delta x(\mathrm{~m})$ along the longitudinal direction of the vehicle at any foot/ soil interface, the final leg swing angle of the leg which slips, needs to be modified in order to achieve the same step size for all the legs.

The final swing angle of the leg, which slips, is deduced as,

$\gamma_{l e g_{-} f n l}^{\prime}=\tan ^{-1}\left(\frac{x_{\text {step_f }} f n l+\Delta x}{L_{3}}\right)$

where, $\gamma_{l e g}^{\prime} f n l$ is the final angle of leg swing due to slip(degree).

With simultaneous level difference of the legs $\left(\Delta h_{l e g}\right)(\mathrm{m})$ and/ or submergence $\left(\Delta h_{\text {foot }}\right)(\mathrm{m})$ and slip $(\Delta x)(\mathrm{m})$, the initial swing angle of the leg concerned will be $\gamma_{l e g \_i n t l} 2$ (degree) and the final swing angle of the leg will be 'ंleg_fnl (degree).

\section{Theoretical Gait Plan for Curvilinear Locomotion}

The principle of skid steering is used for tracked vehicles where one track is driven faster than the other, causing the vehicle to turn towards the slower track. A new principle of skid steering is applied to the designed legged vehicle where differential step sizes are applied to the inner and outer legs as a result of which the vehicle turns towards the inner legs with lower step sizes.

Differential step sizes can be achieved in two ways using 1) inner legs with equal initial swing angles $\left(\gamma /\right.$ leg_intl_in $\neq 0^{\circ}$ ) and outer legs with equal initial swing angles ( $\gamma$ leg_intl_out $\neq 0^{\circ} \quad$ ), where

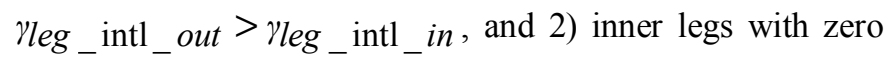
initial swing angles ( $\gamma$ leg intl in $=0^{\circ}$ ) and outer legs with equal initial swing angles $\left(\gamma /\right.$ leg_intl_out $\neq 0^{\circ}$ ). For ease of operation the initial and final swing angles should be equal for all the legs.

The principle of curvilinear locomotion is shown in Figure 4. The two rectangles represent the positions of the main body frame of the vehicle before and after turning. The center of gravity of the vehicle is assumed at the mid-point of the vehicle main body frame. The instantaneous center of rotation is given by the point of intersections of the perpendiculars drawn to the longitudinal center lines of the main body frame at the assumed center of gravity. The turning radius for any point is given by the linear distance between the instantaneous center of rotation and the point under consideration.

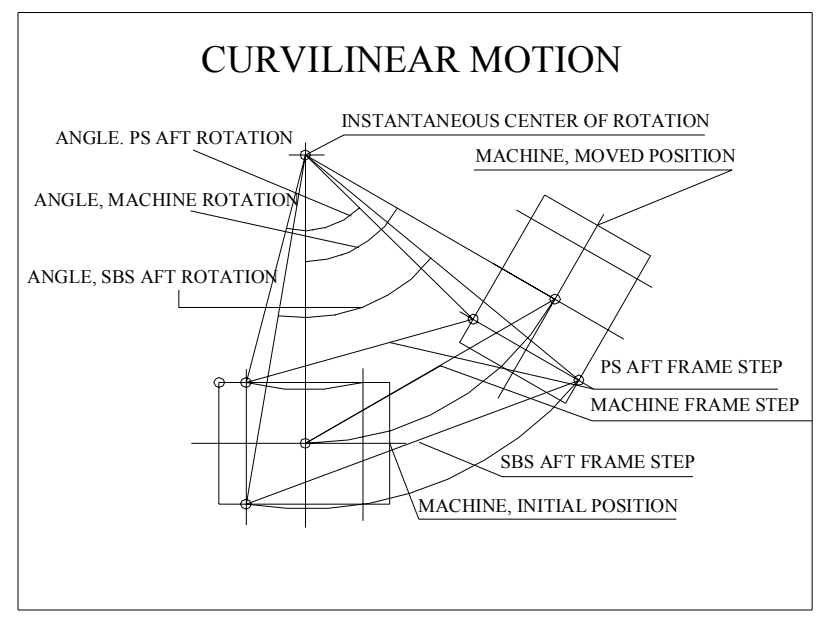

Figure 4. Principle of Curvilinear Locomotion

According to the design, two separate hydraulic circuits are used to drive the inner and outer legs. If the hydraulic oil flows of both the circuits are the same, the time required by the outer legs to swing will be higher than the inner legs. The 
inner legs might slip or skid under the given circumstances. The hydraulic oil flows may be adjusted for the two separate hydraulic circuits, so that both the inner and outer legs have the same swinging time irrespective of the swing angles.

A two-dimensional model for curvilinear locomotion on level and relatively flat terrain was developed. The two dimensional model predicted the turning radius and the yaw velocity of the designed vehicle as a function of the angular velocities of the outer and inner legs.

The turning radius of the vehicle is given as,

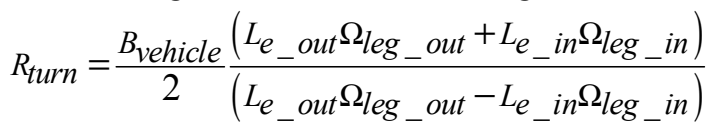

and the yaw velocity of the vehicle is deduced as,

$\Omega_{\text {vehicle }}=\frac{L_{e_{\text {out }}} \Omega_{\text {leg_out }}+L_{e_{\text {in }}} \Omega_{\text {leg_in }}}{2 R_{\text {turn }}}$

where, $R_{\text {turn }}$ is the turning radius of the vehicle $(\mathrm{m})$, $\Omega_{\text {leg out }}$ is the angular velocity of the outer legs ( $\left.\mathrm{rad} / \mathrm{sec}\right)$, $\Omega_{l e g_{\text {in }}}$ is the angular velocity of the inner legs ( $\left.\mathrm{rad} / \mathrm{sec}\right)$, and $L_{e_{-} \text {out }}(\mathrm{m})$ and $L_{e_{-}}$in $(\mathrm{m})$ are the effective leg lengths of the outer and inner legs respectively.

Comparisons between the predicted and experimental results for straight line and curvilinear locomotion are presented next.

\section{COMPARISONS BETWEEN PREDICTED AND EXPERIMENTAL RESULTS}

Full scale on land prototype tests for both forward and backward straight-line locomotion were carried out on level and relatively flat terrain. Prototype tests for validation of the theoretical gait plans for curvilinear locomotion were also executed. Representative results for forward straight line and curvilinear locomotion are presented in this paper. Figure 5 and 6 shows the comparison between the theoretical and experimental step sizes for forward straight-line locomotion. The slip at the foot/ soil interface was not considered.

The prototype vehicle was remotely operated on land by manually controlling the electronic switches actuating the solenoids of the directional control (DC) valves of the hydraulic cylinders. It was thus difficult to accurately control the final swing angle of the legs. Due to human operational errors, the deviations between the experimental and predicted results existed. The deviations between the experimental and predicted results can also be explained due to the presence of the slip at the foot/ soil interfaces.

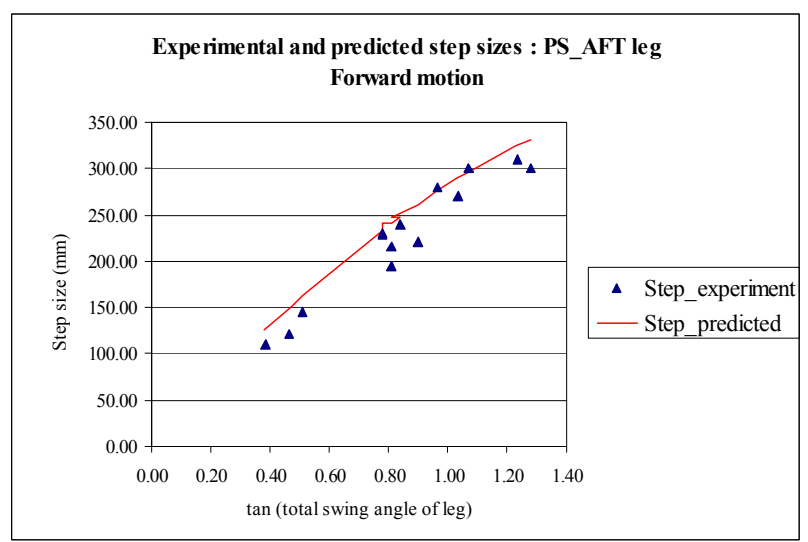

Figure 5. Experimental step size and predicted step size without slip for port side aft leg during forward straight-line locomotion

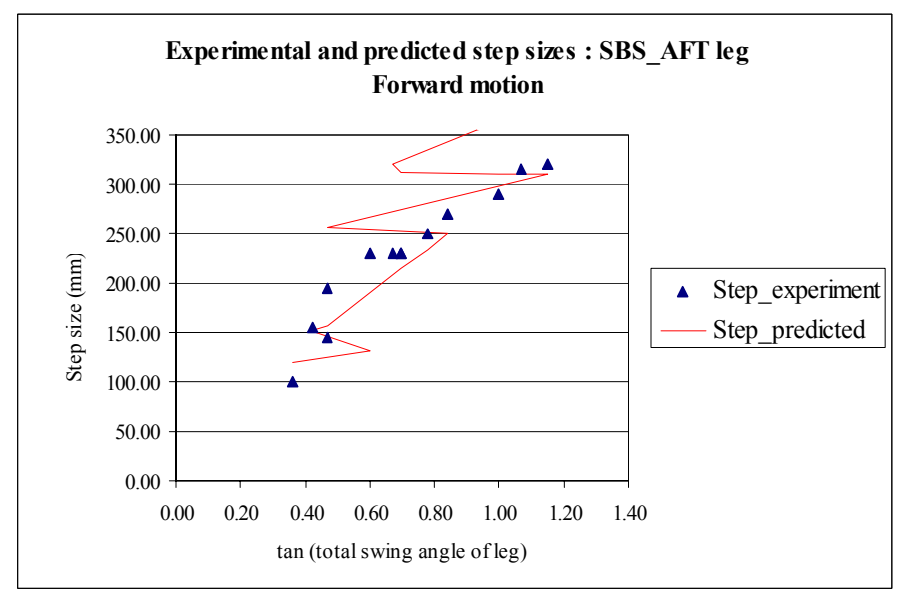

Figure 6. Experimental step size and predicted step size without slip for starboard side aft leg during forward straight-line locomotion

The experimental step sizes for forward straight-line locomotion were next compared with the predicted step sizes, which considered the slip at the foot/ soil interface. The results are shown in Figure 7. Such comparisons help to estimate the slip percentage occurring at the different foot/ soil interfaces for the particular soil on which the locomotion tests were performed. The soil consisted of relatively dry medium to fine sand. The effective leg lengths, the angular and the linear velocities of the legs at different slip percentages were calculated from the experimental data. The step sizes were predicted from the linear velocities of the legs and the time required swinging the leg through the total swing angle. 

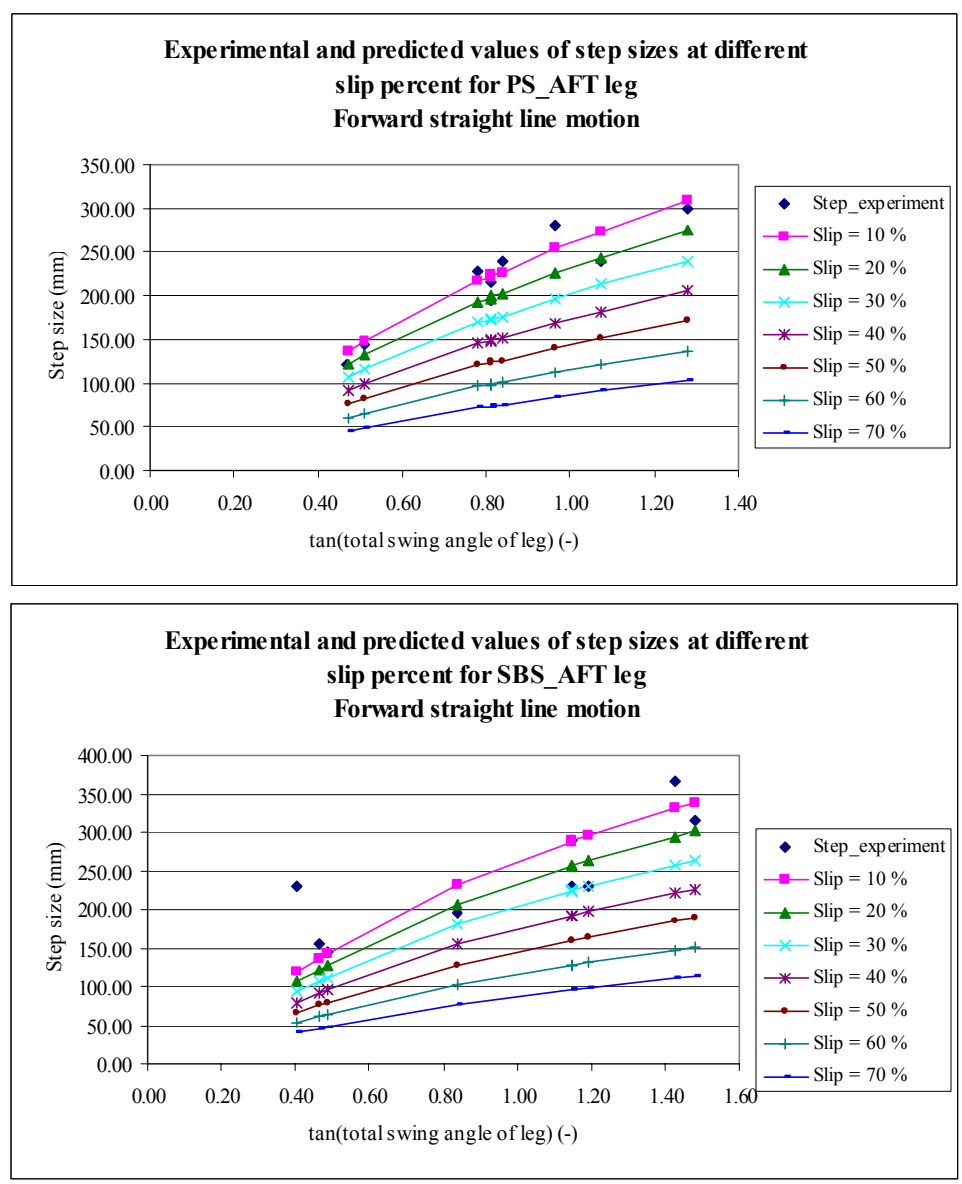

Figure 7. Experimental and predicted step sizes with different slip percentages during forward straight-line locomotion

It is observed from Figure 7, that the experimental step sizes are close to the predicted step sizes with a slip of $10 \%$ for the port side aft leg. In case of the starboard side aft leg, the experimental step sizes are close to the predicted step sizes with slips of $10 \%$ and $20 \%$. The average slip percentage calculated from the experimental results for the port side aft leg was $12 \%$ and that for the starboard side leg was $17 \%$. From the predicted and experimental results it can be concluded that the slip percentage for the designed leg and foot in medium to fine relatively dry sand varies between 10 to $20 \%$ for forward straight-line locomotion. Manual operation of the prototype, presence of the ladder assembly and the lagging and leading effects of the port side and starboard side hydraulic circuits explain the differences between the slip percentages for the port side aft and starboard side aft legs.

Comparisons between the theoretical and experimental results for curvilinear locomotion with unequal leg swing angles for the outer and inner legs are presented next. The turning radius was predicted by neglecting the slip or skid at the foot/ soil interface. The predicted turning radius was compared with the experimental turning radius. The comparisons are shown in Figure 8.

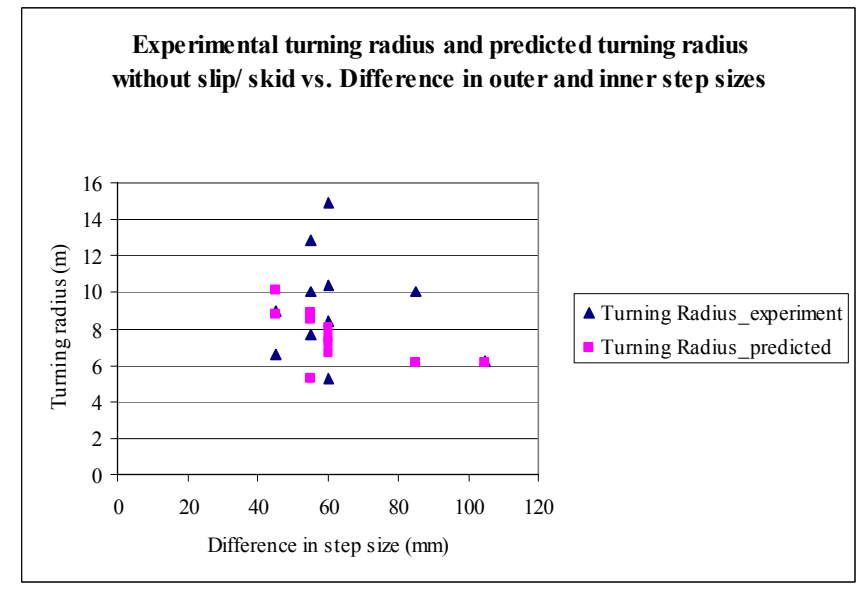

Figure 8. Experimental turning radius and predicted turning radius without slip/ skid at the foot/ soil interface

No correspondence is observed between the experimental and predicted results.

The deviations between the experimental and predicted step sizes are due to 1) manual operation of the electronic switches actuating the solenoids of the directional control valves operating the hydraulic actuators, 2) slip or skid at the foot/ soil interface, and 3) absence of oil flow control system in the designed hydraulic circuits.

Due to manual operation, it was extremely difficult to control the final swing angles of the legs. Sometimes one or more leg was still swinging while the others stopped their movements. The manual operation posed problems in accurately following the theoretical gait plans developed and as a result the vehicle exhibited fairly complex turning behaviors. This resulted in unusual slip or skid actions at the foot/ soil interfaces too. As long as there is a leg swing velocity, the respective foot has a tendency to slip. When there is no swing velocity of the inner legs, the inner feet have tendencies to both slip and skid. This occurs when the inner legs have stopped swinging and the outer legs still exhibit swinging motion. Due to the absence of a flow control system in the hydraulic circuits and unequal leg swing angles of the inner and outer legs, these phenomena will occur frequently leading to deviations between experimental and predicted results. With the present experimental set up, the deviations between the experimental and predicted values are unavoidable but the results do show the general performance of the vehicle.

The turning angle of the vehicle was predicted by neglecting the effect of slip/ skid at the foot/ soil interface. The comparison between the experimental and predicted turning angles of the vehicle is shown in Figure 9.

The vehicle turning angles were very small and hence the deviation observed was also of the order of 0.5 degree. Such deviations can be neglected under the given experimental set up and measuring techniques used. 


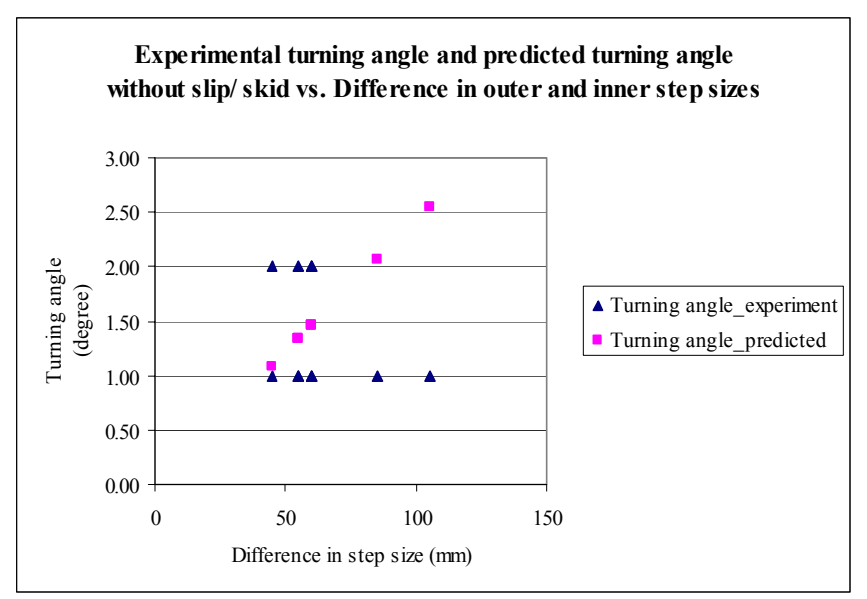

Figure 9. Experimental turning angle and predicted turning angle without slip/ skid at the foot/ soil interface

\section{CONCLUSIONS}

The designed vehicle was able to follow the theoretical gait planners with acceptable accuracy, even with manual operation of the electronic switches of the solenoids of the directional control valves actuating the hydraulic cylinders. Such results show the effectiveness of using the designed leg linkage and the proposed method of locomotion for submersible legged dredgers/ miners. With the development of suitable control system, the legs can be controlled accurately. A slip of 10 to $20 \%$ was observed at the foot/soil interface during forward straight-line locomotion. Comparisons between the theoretical and experimental results for curvilinear locomotion without the consideration of the slip at the foot/ soil interface did not show good correspondence. The gait plans for curvilinear locomotion were modified by considering the slip or skid at the foot/ soil interface, which showed greater correspondence between theoretical and experimental results.

\section{ACKNOWLEDGMENT}

The authors acknowledge the financial support from the Atlantic Innovation Fund Project, Subsea Engineering Underwater operations, robotics and intelligent systems, under the Pan-Atlantic Petroleum Systems Consortium and Memorial University for a graduate fellowship to Ms. S. Sarkar for carrying out this research project. The authors are also thankful to Excavation \& Equipment Manufacturing Pvt. Ltd. (EEM (P) Ltd.), India, for their knowledge exchange, involvement, manufacturing and testing of the full-scale prototype vehicle in their workshop premises. 